\title{
Multi-kink scattering in the double sine-Gordon model
}

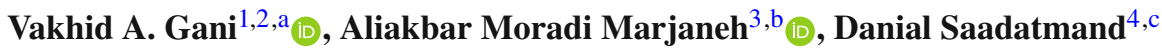 \\ ${ }^{1}$ Department of Mathematics, National Research Nuclear University MEPhI (Moscow Engineering Physics Institute), 115409 Moscow, Russia \\ 2 Theory Department, National Research Center Kurchatov Institute, Institute for Theoretical and Experimental Physics, 117218 Moscow, Russia \\ ${ }^{3}$ Young Researchers and Elite Club, Quchan Branch, Islamic Azad University, Quchan, Iran \\ ${ }^{4}$ Department of Physics, University of Sistan and Baluchestan, Zahedan, Iran
}

Received: 23 January 2019 / Accepted: 10 July 2019 / Published online: 24 July 2019

(C) The Author(s) 2019

\begin{abstract}
We study collisions of two, three, and four kinks of the double sine-Gordon model. The initial conditions are taken in a special form in order to provide collision of all kinks in one point. We obtain dependences of the maximal energy densities on the model parameter. We also analyze the final states observed in these collisions.
\end{abstract}

\section{Introduction}

Interactions of kinks - solutions of the type of solitary waves of non-integrable field-theoretical models in $(1+1)$ dimensional space-time - are of growing interest [1-4]. Great amount of important results has been obtained recently for the kink-(anti)kink scattering [5-16]. In many cases rather non-trivial picture of interaction has been observed. In particular, it was found that the collision of kink and antikink crucially depends on the initial velocity $v_{\text {in }}$ (in the numerical experiments kink and antikink are initially placed at large distance from each other and are moving towards each other with the initial velocities $v_{\text {in }}$ in the laboratory frame of reference). There is a critical value of the initial velocity $v_{\mathrm{cr}}$, which separates two different regimes of the collision. At $v_{\text {in }}>v_{\text {cr }}$ inelastic reflection of kinks (or passing through each other, if the model allows such process) is observed. At $v_{\text {in }}<v_{\text {cr }}$ after the collision the kink and antikink form a long-living bound state - a bion - which decays slowly radiating energy in the form of small-amplitude waves. Besides, in some models at $v_{\text {in }}<v_{\text {cr }}$ an interesting phenomenon was found - so-called escape windows. Escape windows are intervals of the initial velocity, at which kinks do not form a bion, but escape from each other after two or more collisions. The cause of this phenomenon is the resonance energy exchange between

\footnotetext{
a e-mail: vagani@mephi.ru

be-mail: moradimarjaneh@gmail.com

c e-mail: saadatmand.d@gmail.com
}

translational and vibrational modes of the kink (antikink), see, e.g., Refs. [17-19]. In some models the kinetic energy of the kinks can be stored in vibrational modes of the system "kink + antikink" [20-23].

Another very interesting subject that should be mentioned here is interactions of kinks having power-law tails, see, e.g., Refs. [22-30]. Such kinks arise in various models with both polynomial and non-polynomial potentials. The power-law asymptotics ("fat tails") of kinks result in their long-range interaction, i.e. the kinks feel each other at very large distances. As a result, the traditionally used initial conditions in the form of superposition of kink and antikink seem to be not valid for simulation of the kink-antikink scattering [26].

The interactions of kinks are studied both numerically and analytically. On the one hand, the scattering processes can be simulated directly by using the numerical methods of solving equations of motion - partial differential equations of second order. On the other hand, there are analytical approximate methods which allow to estimate forces between kink and (anti)kink. These are (i) the collective coordinate approximation [5,31,32] and (ii) Manton's method [3, Ch. 5] [3336].

It is worth to mention that kink-like solutions can also be obtained in more complicated models with two or more scalar fields [37-53]. In particular, in [37,38,41,47-52] the models with two real scalar fields with polynomial potentials have been studied. In [54-60] configurations of the type of domain wall with additional fields localized on it were investigated. Many important results have also been obtained for non-topological field configurations - lumps, Q-balls, etc. [61-77].

Kinks, domain walls and other topological defects arise in a great amount of models, hence they are very important for various physical applications from condensed matter to high energy physics and cosmology [78-85], see also [2]. Many physical phenomena could be effectively described 
Fig. 1 a Potential of the DSG model; $\mathbf{b} R$-dependence of the coefficients in the potential, which are responsible for the mixing of two sine-Gordon potentials

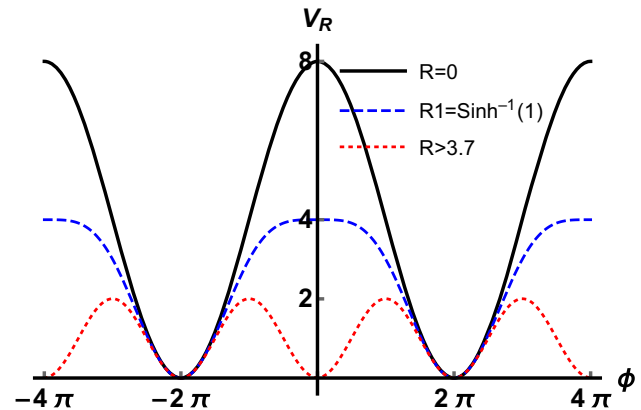

(a)

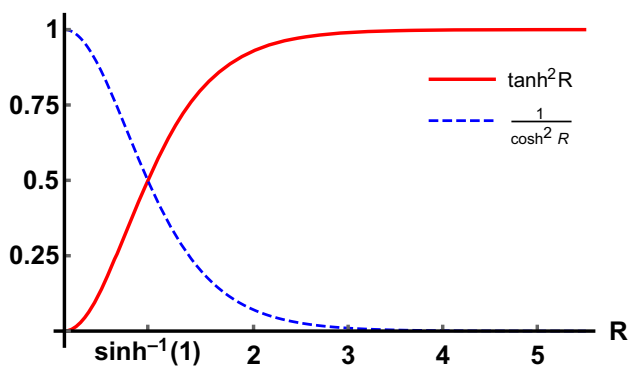

(b) by one-dimensional topological structures. For example, a three-dimensional domain wall in the direction perpendicular to it can be viewed as a one-dimensional topological field configuration - kink connecting two different vacua of the model. At the same time, the case of $(1+1)$-dimensional space-time structure can be investigated more easily compared to $2+1$ or $3+1$ dimensions.

The processes of collision of several kinks in one point (with extracting maximal energy densities) have been actively investigated over the last few years [86-90]. Such processes are of interest for numerous applications. Being topological defects, the kinks bear significant amount of energy. As a result, simultaneous collision of several kinks (antikinks) in one point can lead to formation of spatial domains with high energy density. The authors of Ref. [90] have shown that with knowing maximal energy densities in collision of $N$ kinks one can predict how many kink-antikink pairs can be produced in particle collisions.

This study deals with the collisions of several kinks of the double sine-Gordon (DSG) model in one point. This model has been actively studied due to its numerous applications, see, e.g., [10,20,91-93] and references therein. Note that the subject of multi-kink collisions is very vast. The number of parameters that could be changed is very large. These are, in particular, the model parameter $R$, the initial velocities and the initial positions of kinks. In this paper we study collisions of two three and four kinks in one point (in a small spatial region). We focus on the dependences of maximal energy densities on the model parameter $R$. In collisions of four kinks (two kinks and two antikinks, to be more accurate) we investigate how the final configurations depend on $R$. In our numerical simulations we use fixed initial positions and initial velocities of kinks.

Our paper is organized as follows. In Sect. 2 we recall the main features of the DSG model, write down its kinks, and discuss their main properties. Section 3 presents the results of the numerical simulations of multi-kink collisions. We conclude with summarizing in Sect. 4 , where we also formulate some possible directions for further research.

\section{Double sine-Gordon model}

Within the $(1+1)$-dimensional double sine-Gordon model the dynamics of the real scalar field $\phi(x, t)$ is described by the Lagrangian density

$\mathcal{L}=\frac{1}{2}\left(\frac{\partial \phi}{\partial t}\right)^{2}-\frac{1}{2}\left(\frac{\partial \phi}{\partial x}\right)^{2}-V(\phi)$

where the potential $V(\phi)$ is non-negative function of the field. From the Lagrangian (1) one can obtain the equation of motion for the field $\phi(x, t)$ :

$\frac{\partial^{2} \phi}{\partial t^{2}}-\frac{\partial^{2} \phi}{\partial x^{2}}+\frac{d V}{d \phi}=0$

Below we simulate multi-kink scattering by solving this equation numerically.

In literature there are various modifications and parameterizations of the potential, which all can be called "double sine-Gordon potential". We will use the so-called $R$ parameterization, see, e.g., Refs. [10,20,91]. In this parameterization the DSG potential is:

$V(\phi)=\tanh ^{2} R(1-\cos \phi)+\frac{4}{\cosh ^{2} R}\left(1+\cos \frac{\phi}{2}\right)$.

In Fig. 1a this potential for some values of the parameter and Fig. $1 \mathrm{~b}$ the $R$-dependence of the coefficients in front of $(1-\cos \phi)$ and $\left(1+\cos \frac{\phi}{2}\right)$, which are responsible for the "mixing" of $\phi$ - and $\frac{\phi}{2}$-sine-Gordon potentials. Depending on $R$, the potential (3) looks different. In particular,

$V(\phi)=\left\{\begin{array}{l}4\left(1+\cos \frac{\phi}{2}\right) \text { for } R=0, \\ 4-\left(1-\cos \frac{\phi}{2}\right)^{2} \text { for } R=\operatorname{arsinh} 1, \\ 1-\cos \phi \text { for } R \rightarrow+\infty .\end{array}\right.$ 


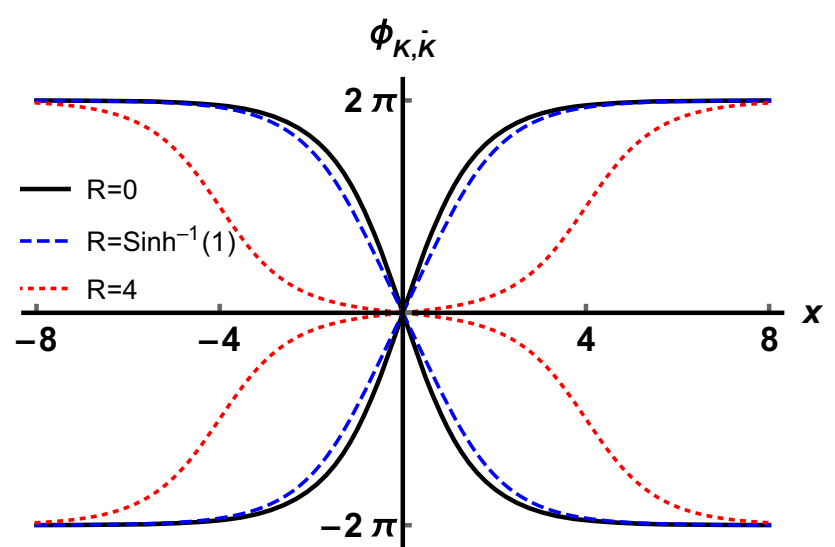

Fig. 2 Kinks (monotonously increasing functions $\phi_{\mathrm{k}}(x)$ ) and antikinks (monotonously decreasing functions $\phi_{\overline{\mathrm{k}}}(x)$ ) of the DSG model for three different values of the parameter $R$

The double sine-Gordon model has static topological solution - kink (antikink):

$\phi_{\mathrm{k}(\overline{\mathrm{k}})}(x)=4 \pi n \pm 4 \arctan \frac{\sinh x}{\cosh R}$.

Notice that for better understanding of many processes in kinks collisions the following fact could be important: the DSG kink (antikink) (5) can be interpreted as a superposition of two sine-Gordon solitons:

$\phi_{\mathrm{k}(\overline{\mathrm{k}})}(x)=4 \pi n \pm\left[\phi_{\mathrm{SGK}}(x+R)-\phi_{\mathrm{SGK}}(R-x)\right]$,

where

$\phi_{\mathrm{SGK}}(x)=4 \arctan \exp (x)$

is the sine-Gordon kink (soliton). Thus the DSG kink can be viewed as a superposition of two sine-Gordon kinks, which are separated by the distance $2 R$ (if the DSG kink is centered at $x=0$ then the two sine-Gordon solitons are centered at $x= \pm R$ ), see Fig. 2. Due to the Lorentz invariance, the kink (antikink) moving along the $x$-axis with the velocity $v$ can be obtained by the Lorentz boost:

$\phi_{\mathrm{k}(\overline{\mathrm{k}})}(x, t)=\phi_{\mathrm{k}(\overline{\mathrm{k}})}(\gamma(x-v t))$,

where $\gamma=1 / \sqrt{1-v^{2}}$ is the Lorentz factor.

The energy functional which corresponds to the Lagrangian (1) reads

$E[\phi]=\int_{-\infty}^{\infty}\left[\frac{1}{2}\left(\frac{\partial \phi}{\partial t}\right)^{2}+\frac{1}{2}\left(\frac{\partial \phi}{\partial x}\right)^{2}+V(\phi)\right] d x$.

The total energy (9) can be split into three parts: (i) the kinetic energy $K$, (ii) the gradient energy $U$, and (iii) the potential energy $P$ :
$E=K+U+P$.

According to this, the integrand in (9), i.e. the total energy density $\varepsilon(x, t)$, can be written as

$\varepsilon(x, t)=k(x, t)+u(x, t)+p(x, t)$,

where

$k(x, t)=\frac{1}{2}\left(\frac{\partial \phi}{\partial t}\right)^{2}, \quad u(x, t)=\frac{1}{2}\left(\frac{\partial \phi}{\partial x}\right)^{2}$,

$p(x, t)=V(\phi)$

are (i) the kinetic energy density, (ii) the gradient energy density, and (iii) the potential energy density, respectively.

In the case of one moving kink $\phi_{\mathrm{k}}(x, t)$ we have:

$$
\begin{aligned}
p(x, t) & =\frac{8 \cosh ^{2} R \cdot \cosh ^{2}[\gamma(x-v t)]}{\left(\cosh ^{2} R+\sinh ^{2}[\gamma(x-v t)]\right)^{2}} \\
& =\left(1-v^{2}\right) u(x, t)=\frac{1-v^{2}}{v^{2}} k(x, t) \\
& =\frac{1-v^{2}}{2} \varepsilon(x, t) .
\end{aligned}
$$

Integrating the total energy density with respect to $x$, we obtain the total energy of the moving kink:

$E_{\mathrm{K}}=\int_{-\infty}^{+\infty} \varepsilon(x, t) d x=\frac{M_{\mathrm{K}}}{\sqrt{1-v^{2}}}$

where

$M_{\mathrm{K}}=16\left(1+\frac{2 R}{\sinh 2 R}\right)$

is the mass of kink, i.e. the energy of the static DSG kink.

In the next section we present the results of the numerical simulation of collisions of two, three and four DSG kinks in the same point. We find the maximal (over the spatial coordinate $x$ and time $t$ ) values of the energy densities mentioned above: kinetic, gradient, potential and total. Note that for one moving kink the maximal values can be obtained analytically. Depending on the parameter $R$, they are the following:

$$
\begin{aligned}
p_{\max }^{(1)} & =\left(1-v^{2}\right) u_{\max }^{(1)}=\frac{1-v^{2}}{v^{2}} k_{\max }^{(1)}=\frac{1-v^{2}}{2} \varepsilon_{\max }^{(1)} \\
& =\frac{8}{\cosh ^{2} R}
\end{aligned}
$$

at $R \leq \operatorname{arsinh} 1$, and

$$
\begin{aligned}
p_{\max }^{(1)} & =\left(1-v^{2}\right) u_{\max }^{(1)}=\frac{1-v^{2}}{v^{2}} k_{\max }^{(1)}=\frac{1-v^{2}}{2} \varepsilon_{\max }^{(1)} \\
& =2 \operatorname{coth}^{2} R
\end{aligned}
$$

at $R \geq \operatorname{arsinh} 1$. 


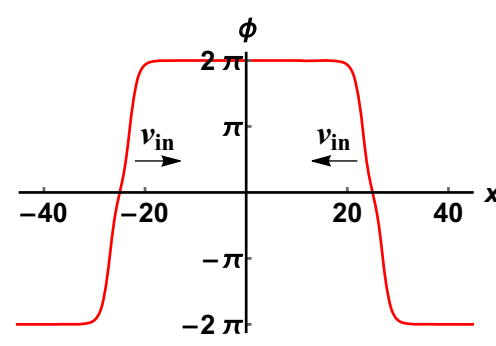

(a) kink+antikink

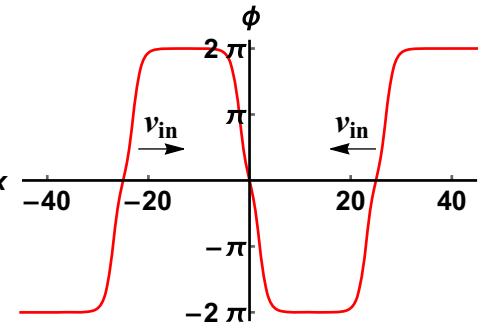

(b) kink+antikink+kink

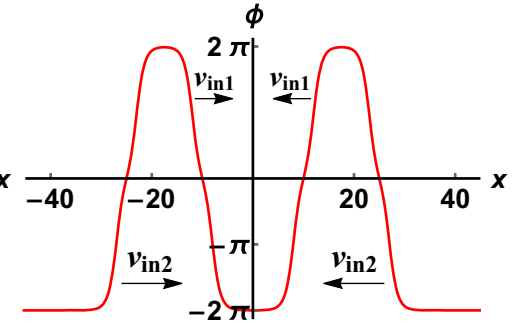

(c) kink+antikink + kink+antikink

Fig. 3 The initial configurations for $R=2$ : a Eq. (19), b Eq. (20), c Eq. (21)

\section{Multi-kink scattering}

We studied the collisions of two kinks (a kink and an antikink), three kinks (two kinks and an antikink), and four kinks (two kinks and two antikinks) at one point. Our main goal was to obtain $R$-dependences of the maximal values of the energy densities - kinetic, gradient, potential and total. To do this, we numerically solved the discretized version of the equation of motion (2),

$$
\begin{aligned}
& \frac{d^{2} \phi_{n}}{d t^{2}}-\frac{1}{h^{2}}\left(\phi_{n-1}-2 \phi_{n}+\phi_{n+1}\right) \\
& +\frac{1}{12 h^{2}}\left(\phi_{n-2}-4 \phi_{n-1}+6 \phi_{n}-4 \phi_{n+1}+\phi_{n+2}\right) \\
& +\tanh ^{2} R \sin \phi_{n}-\frac{2}{\cosh ^{2} R} \sin \frac{\phi_{n}}{2}=0,
\end{aligned}
$$

using Störmer method of integration. Here $h=0.025$ is the space step, $n=0, \pm 1, \pm 2, \ldots$ and $\phi_{n}(t)=\phi(n h, t)$. The second derivative of the field $\phi$ with respect to $x, \phi_{x x}$, is discretized with the accuracy $O\left(h^{4}\right)$. In our numerical experiments we used the range $-375 \leq x \leq 375$. Integration with respect to time was performed with the step $\tau=0.005$. We used $0 \leq t \leq 500$ for searching maximal energy densities over $x$ and $t$.

The initial conditions for each case (collisions of two, three and four kinks) will be written below. In the case of collisions of three and four kinks, the initial conditions were fit in such a way that the kinks collided in one point.

From the point of view of physical applications, the energy distribution in collision of two or several kinks is of great importance. The point is that a kink is a topological defect, which describes, for example, a planar domain wall or another formation ("defect") in a real physical system. The kinks collision thus represents mathematical model of collision of such defects, and the energy densities show space-time distribution of energy.

\subsection{Kink-antikink scattering}

First of all, note that collisions of the DSG kink and antikink have been studied in detail in recent paper [10]. An interesting phenomenon was found - oscillons in the final state. Besides, the dependence of the critical velocity $v_{\text {cr }}$ on $R$ obtained in [10] was somewhat different from that presented in classical paper [91]. We think that a series of local maxima of the dependence $v_{\mathrm{cr}}(R)$ observed in [10] is a consequence of more detailed investigation of appropriate range of the initial velocities of the colliding kinks.

Unlike the previous works $[10,20,91]$, we focus on study of maximal energy densities in collisions of the kink and antikink at the initial velocity $v_{\text {in }}=0.1$ and various $R$. To do this we used the initial condition in the form of the kink and antikink, which are initially placed at $x=-x_{0}$ and $x=+x_{0}$ and moving towards each other with the velocities $v=+v_{\text {in }}$ and $v=-v_{\text {in }}$, respectively,

$$
\begin{aligned}
\phi_{\mathrm{k} \overline{\mathrm{k}}}(x)= & \phi_{\mathrm{k}}\left(\frac{x+x_{0}-v_{\mathrm{in}} t}{\sqrt{1-v_{\mathrm{in}}^{2}}}\right) \\
& +\phi_{\overline{\mathrm{k}}}\left(\frac{x-x_{0}+v_{\mathrm{in}} t}{\sqrt{1-v_{\mathrm{in}}^{2}}}\right)-2 \pi
\end{aligned}
$$

where $\phi_{\mathrm{k}(\overline{\mathrm{k}})}(x)$ is defined by Eq. (5) with $n=0$. This initial configuration is shown in Fig. 3a. In our simulations we used $x_{0}=25$ and $v_{\text {in }}=0.1$. Space-time picture of the collision for some selected values of $R$ is shown in Fig. 4.

We performed numerical simulation of the kink-antikink collisions within a wide range of the parameter $R$. We have obtained the dependences of the maximal energy densities on the parameter $R$, see Fig. 5. These dependences are rather complicated. At $R \lesssim 0.5$ and $R \gtrsim 2.5$ we see smooth and monotonously decreasing curves. At the same time, within the range $0.5 \lesssim R \lesssim 2.5$ the dependences look quite stochastic. The observed difference in behavior of the maximal energy densities at various $R$ could be a consequence of different character of kink-antikink interaction at given initial conditions. In particular, depending on the parameter $R$, the initial velocity $v_{\text {in }}=0.1$ can be either less or more than the critical value $v_{\text {cr }}$. The point is that the critical velocity depends on $R$ in a complicated way [10]. 


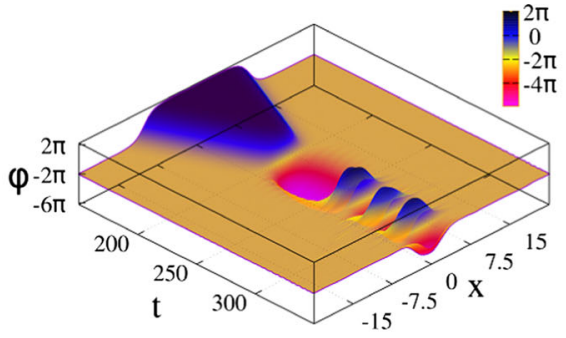

(a) $R=0.5$

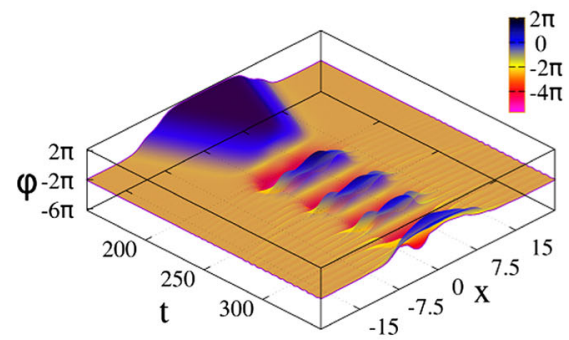

(d) $R=2.0$

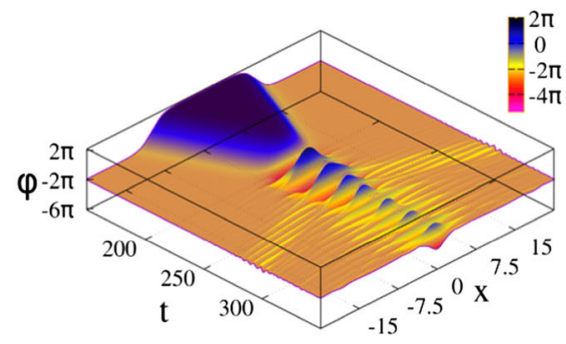

(b) $R=1.0$

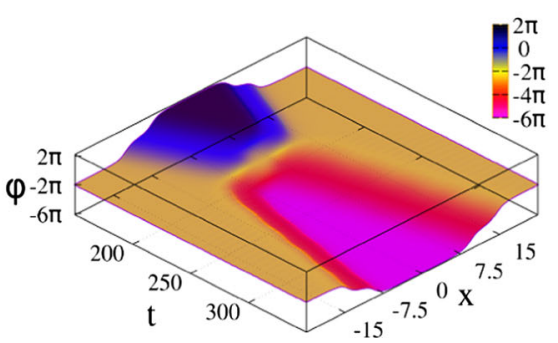

(e) $R=2.5$

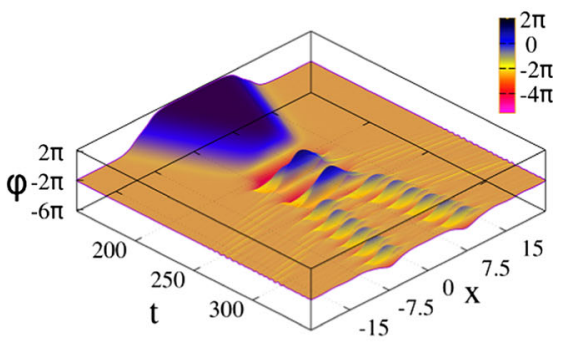

(c) $R=1.5$

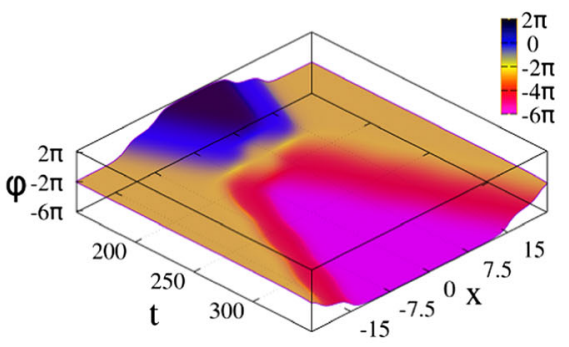

(f) $R=3.0$

Fig. 4 Space-time picture of the kink-antikink collisions for different $R$ 's

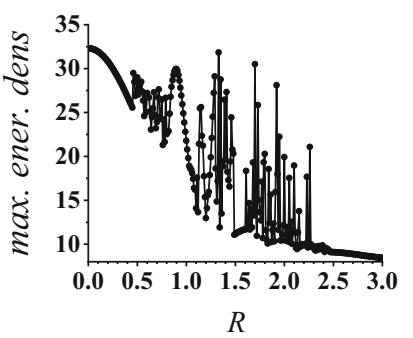

(a) total

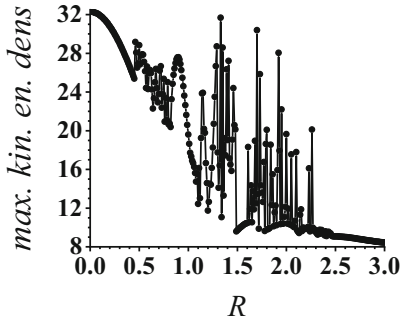

(b) kinetic

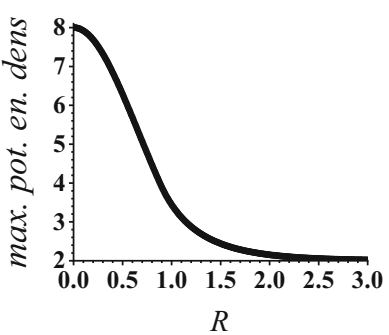

(c) potential

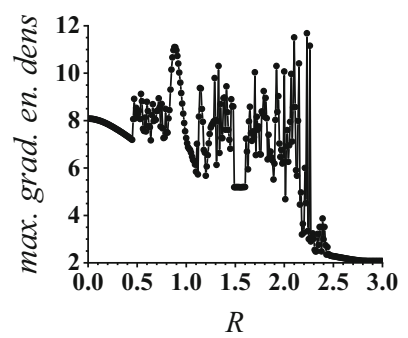

(d) gradient

Fig. 5 Maximal energy densities as functions of $R$ for kink-antikink collisions at $v_{\text {in }}=0.1$

Analyzing the dependence $v_{\mathrm{cr}}(R)$ which is presented in Fig. 6 of Ref. [10], we can see that the initial velocity $v_{\text {in }}=0.1$ is less than the critical in the range $0.5 \lesssim R \lesssim 2.5$. Thus complicated and almost stochastic behavior of the maximal energy densities is observed for those $R$ 's at which the kinks form a bound state, i.e., a bion. On the other hand, at those $R$ 's at which the kinks escape from each other after a collision, the smooth and monotonous dependences are observed. We can assume that the complicated dependences of the maximal energy densities on $R$ can be a consequence of complex resonance processes in bion. As a result, energy is redistributed that leads to abrupt changes of the maximal energy densities.

In order to confirm our hypothesis, we performed numerical simulations of the kink-antikink collisions at $v_{\text {in }}=0.17$ and $v_{\text {in }}=0.2$. The maximal energy densities are shown in Figs. 6 and 7. From these figures one can see that regions of the stochastic behavior of the maximal energy densities become narrower. As it was for $v_{\text {in }}=0.1$, at these new initial velocities the stochastic behavior of the energy densities appears at those $R$ 's at which $v_{\text {in }}<v_{\text {cr }}(R)$ and the kink and antikink capture each other and form a bion in the final state. Another interesting fact is the presence of smooth and monotonous segments of the maximal energy densities in the range $1.5 \lesssim R \lesssim 1.6$, see Fig. 5 . We can assume that it is a consequence of the fact that at these $R$ 's the bound state of kinks evolves differently. The space-time picture of the kink-antikink collision at some selected values of the parameter $R$ from the range $1.49 \leq R \leq 1.63$ is shown in Fig. 8. It it clearly seen that at $1.5 \lesssim R \lesssim 1.6$ two escaping oscillons in the final state are formed after two oscillations of the kink-antikink bound state. At other values of $R$ in Fig. 8 the oscillons in the final state are either formed after more complicated evolution of the kink-antikink bound state or not formed at all. 


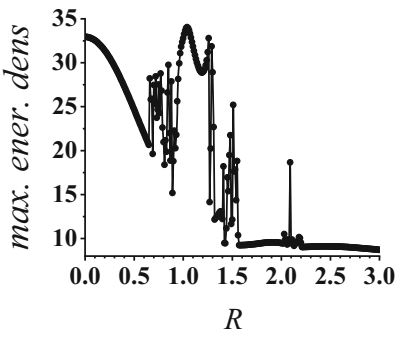

(a) total

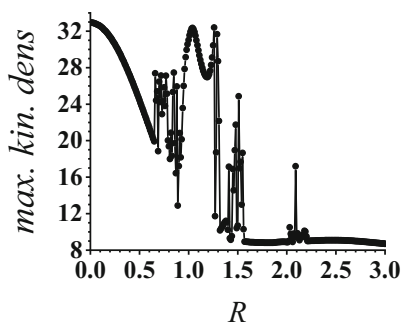

(b) kinetic

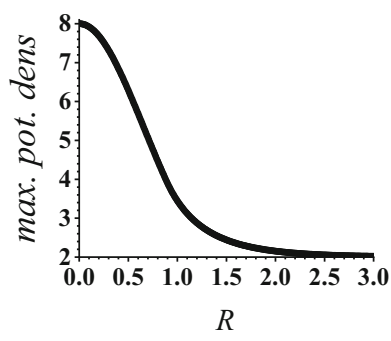

(c) potential

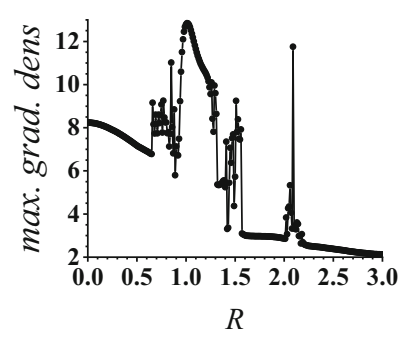

(d) gradient

Fig. 6 Maximal energy densities as functions of $R$ for kink-antikink collisions at $v_{\text {in }}=0.17$

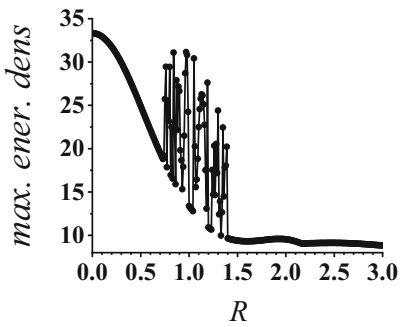

(a) total

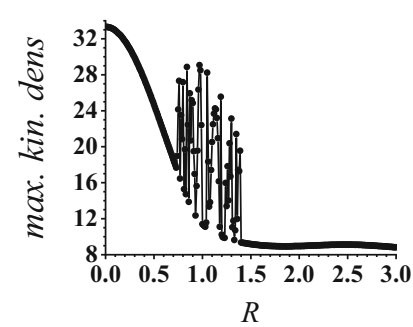

(b) kinetic

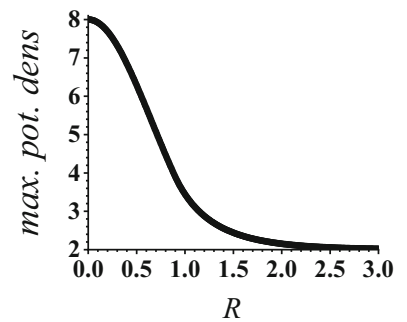

(c) potential

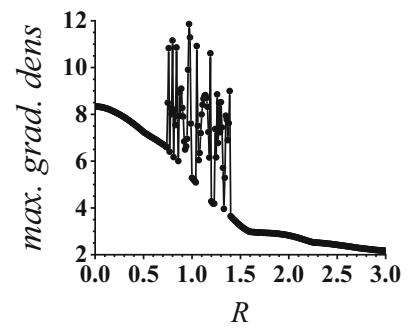

(d) gradient

Fig. 7 Maximal energy densities as functions of $R$ for kink-antikink collisions at $v_{\text {in }}=0.2$

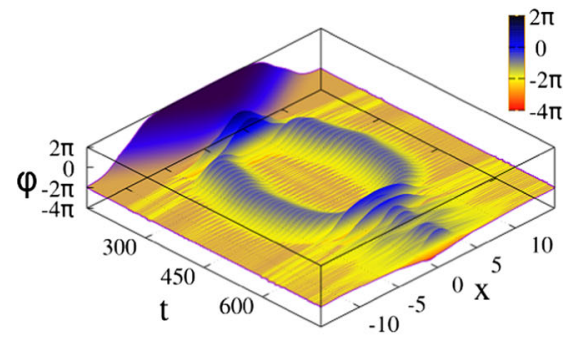

(a) $R=1.49$

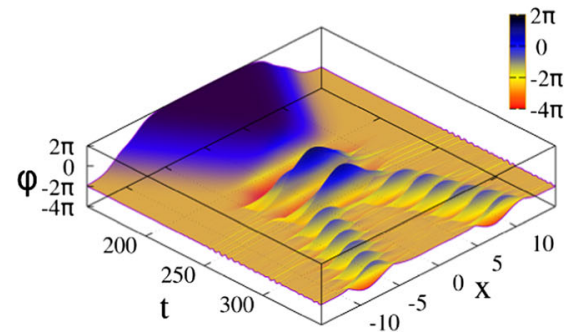

(d) $R=1.60$

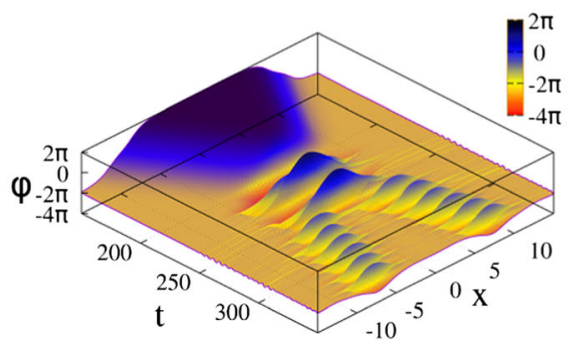

(b) $R=1.50$

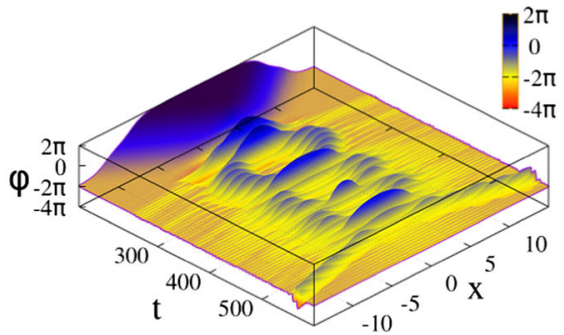

(e) $R=1.61$

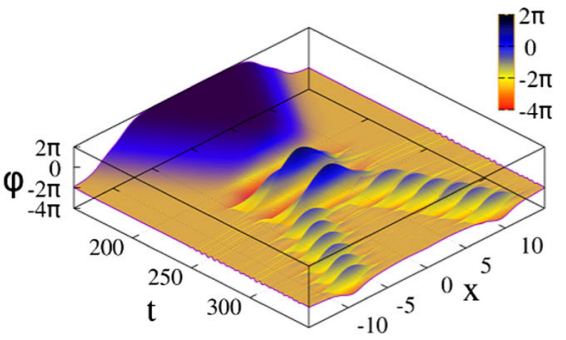

(c) $R=1.52$

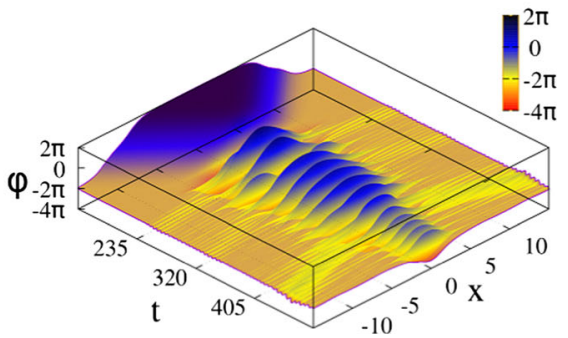

(f) $R=1.63$

Fig. 8 Space-time picture of the kink-antikink collisions for some $R$ 's from the range $1.49 \leq R \leq 1.63$

\subsection{Collision of two kinks and an antikink}

We performed numerical simulation of the collision of three DSG kinks in one point. We used the following initial configuration:

$$
\begin{aligned}
\phi_{\mathrm{k} \overline{\mathrm{k}}}(x)= & \phi_{\mathrm{k}}\left(\frac{x+x_{0}-v_{\mathrm{in}} t}{\sqrt{1-v_{\mathrm{in}}^{2}}}\right)+\phi_{\overline{\mathrm{k}}}(x) \\
& +\phi_{\mathrm{k}}\left(\frac{x-x_{0}+v_{\mathrm{in}} t}{\sqrt{1-v_{\mathrm{in}}^{2}}}\right),
\end{aligned}
$$




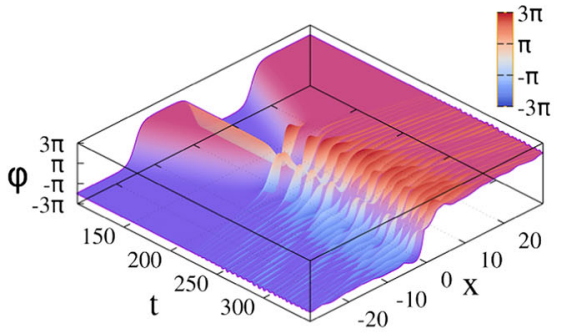

(a) $R=0.50$

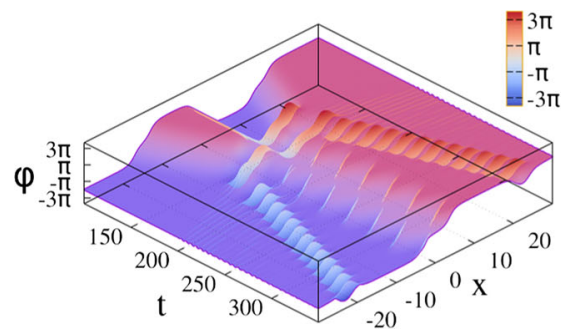

(d) $R=2.0$

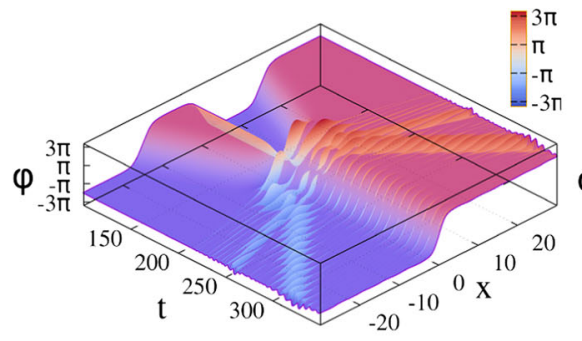

(b) $R=1.0$

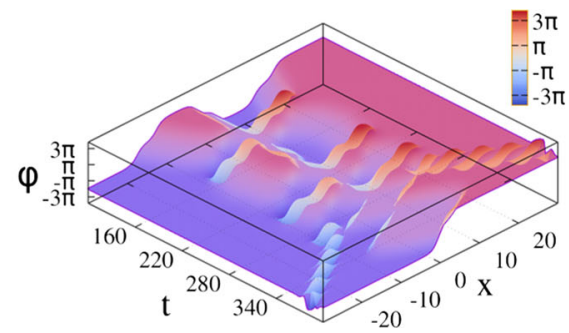

(e) $R=2.5$

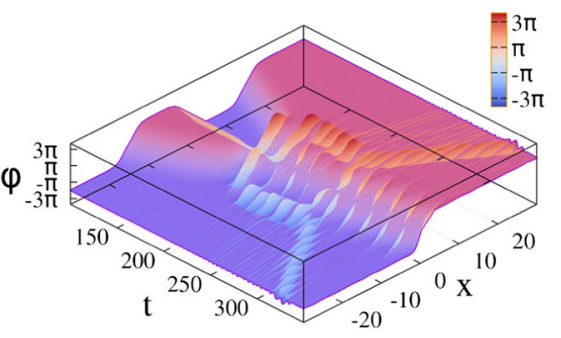

(c) $R=1.5$

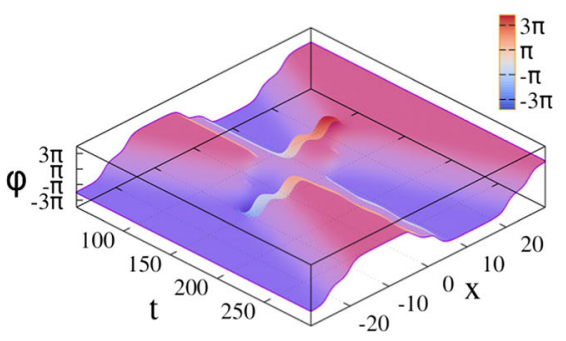

(f) $R=3.0$

Fig. 9 Space-time picture of collisions of three kinks for different $R$ 's

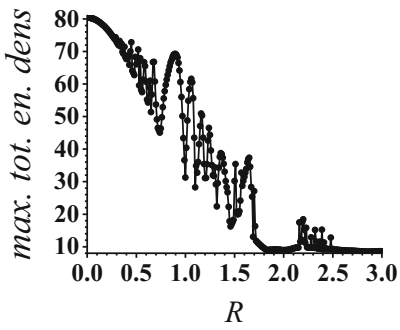

(a) total

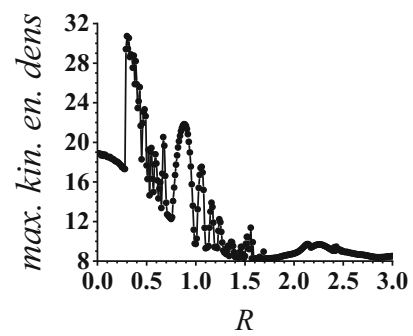

(b) kinetic

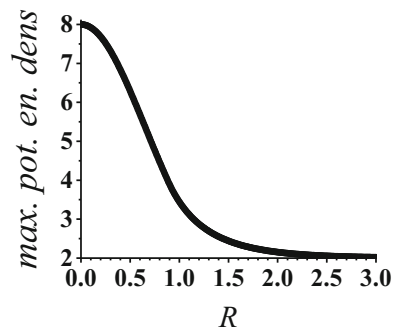

(c) potential

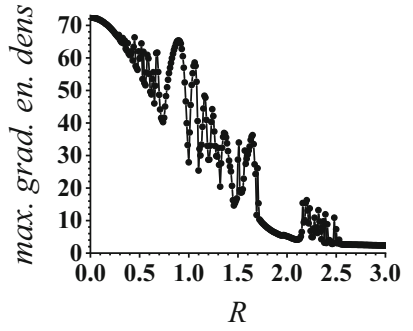

(d) gradient

Fig. 10 Maximal energy densities as functions of $R$ for collisions of three kinks

where $\phi_{\mathrm{k}(\overline{\mathrm{k}})}(x)$ is defined by Eq. (5) with $n=0$. This initial condition corresponds to two kinks and an antikink. The antikink is at rest at the origin $x=0$, and two kinks are moving towards the antikink with the initial velocities $v=+v_{\text {in }}$ and $v=-v_{\text {in }}$, starting from $x=-x_{0}$ and $x=+x_{0}$, respectively. This initial configuration is shown in Fig. 3b. In our simulations we used $x_{0}=25$ and $v_{\text {in }}=0.1$. Space-time picture of the collision for some selected values of $R$ is shown in Fig. 9. The interaction of three kinks looks rather complicated. In particular, in a pair "kink+antikink" resonance interaction can occur. This leads to appearance of various intermediate and final states, see Fig. 9. (Note that the term "final state" stands here for configuration observed after a long time after the collision. "Long time", in turn, means a period of time which is much longer than life-times of various bound states (of oscillons, of kink and oscillon, and so on), if such states do not have infinite life-times.)

We have obtained the dependencies of the maximal energy densities on the parameter $R$, see Fig. 10 . The maximal poten- tial energy density is monotonously decreasing, while the maximal kinetic, gradient and total energy densities behave in a complicated way, thereby reflecting complex interaction of the three colliding kinks.

Note that in the range $1.7 \lesssim R \lesssim 2.1$ we see a smooth and monotonous segments of the maximal energy densities. We can assume that in this range of $R$ the field evolution is somewhat different. Actually, from Fig. 11 we see that at these $R$ 's two escaping oscillons in the final state are formed right after the kinks collision. At the same time, at $R \lesssim 1.7$ and $R \gtrsim 2.1$ the escape of oscillons occur after some time during which the oscillons remain bound.

\subsection{Collision of two kinks and two antikinks}

Finally, we studied the collision of four DSG kinks in one point. To do this, we used the initial configuration of the form of 


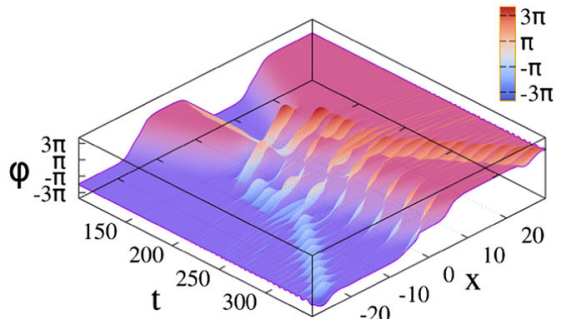

(a) $R=1.60$

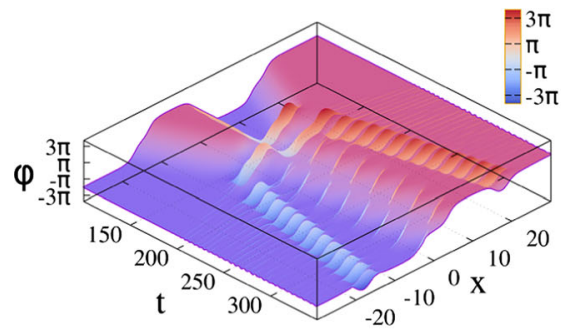

(d) $R=1.86$

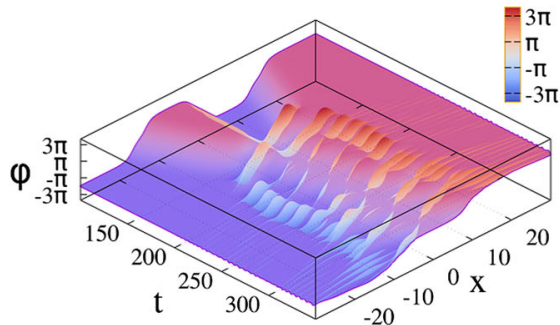

(b) $R=1.70$

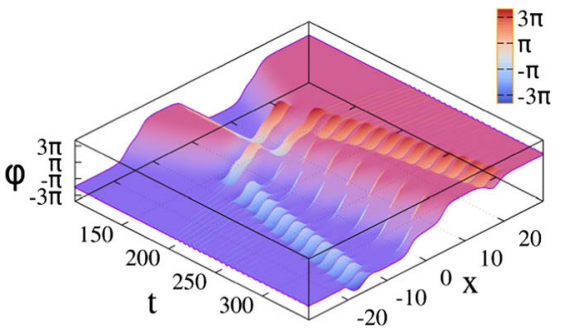

(e) $R=1.94$

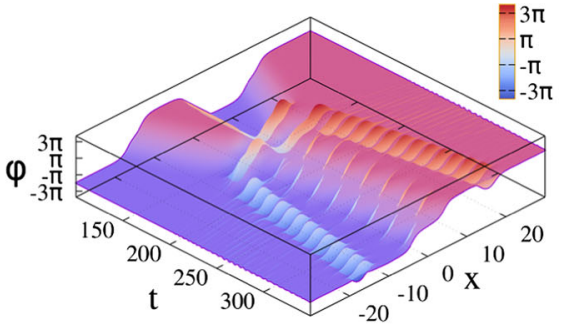

(c) $R=1.78$

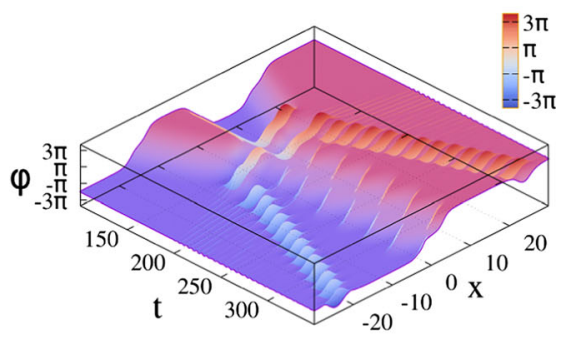

(f) $R=2.02$

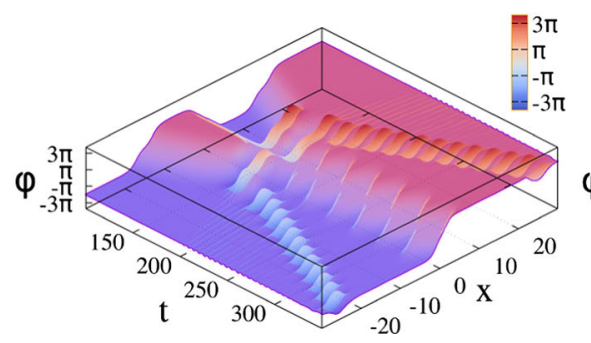

(g) $R=2.10$

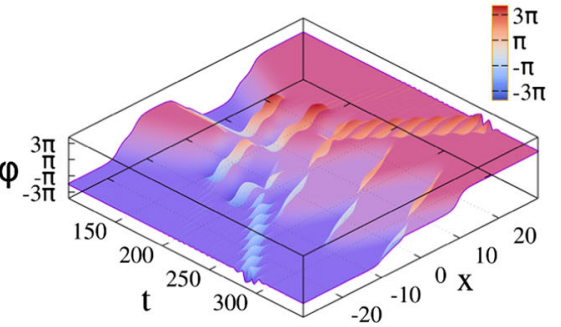

(h) $R=2.20$

Fig. 11 Space-time picture of collisions of three kinks for some $R$ 's from the range $1.6 \leq R \leq 2.2$

$$
\begin{aligned}
\phi_{\mathrm{k} \overline{\mathrm{k}} \overline{\mathrm{k}}}(x)= & \phi_{\mathrm{k}}\left(\frac{x+x_{02}-v_{\mathrm{in} 2} t}{\sqrt{1-v_{\mathrm{in} 2}^{2}}}\right) \\
& +\phi_{\overline{\mathrm{k}}}\left(\frac{x+x_{01}-v_{\mathrm{in} 1} t}{\sqrt{1-v_{\mathrm{in} 1}^{2}}}\right) \\
& +\phi_{\mathrm{k}}\left(\frac{x-x_{01}+v_{\mathrm{in} 1} t}{\sqrt{1-v_{\mathrm{in} 1}^{2}}}\right) \\
& +\phi_{\overline{\mathrm{k}}}\left(\frac{x-x_{02}+v_{\mathrm{in} 2} t}{\sqrt{1-v_{\mathrm{in} 2}^{2}}}\right)-2 \pi,
\end{aligned}
$$

where $\phi_{\mathrm{k}(\overline{\mathrm{k}})}(x)$ is defined by Eq. (5) with $n=0$. The initial configuration (21) corresponds to two kinks and two antikinks moving to the collision point, see Fig. 3c. We used the following initial values of positions and velocities of the kinks and antikinks: $x_{01}=10, x_{02}=25, v_{\text {in } 1}=0.05$, $v_{\text {in2 }}=0.1$. This choice of the parameters guarantees almost simultaneous collision of all four waves in the origin $x=0$.
The space-time picture of the collision for some selected values of the parameter $R$ is presented in Fig. 12. We can see a vast variety of final states. At $R=1.5, R=2.0$ and $R=2.5$ we observe four oscillons in the final state. We could propose the following explanation of the formation of oscillons. Each DSG kink/antikink consists of two subkinks, see Eq. (6). In the kinks collision the constituent subkinks interact with each other in a complicated way, thus forming bound states - oscillons. This means that we consider an oscillon as a bound state of subkinks (at the same time, remind that a bion is a bound state of kink and antikink). At $R=0.5$ we observe formation of two bions. At $R=1.0$ the final state has the form of escaping kink and antikink together with two oscillons also escaping from the collision point. At $R=3.0$ in the final state we see a bion localized near the origin $x=0$ and escaping DSG kink and antikink. Maximal values of the energy densities in the case of four kinks collisions are highly dependent on the initial positions of the kinks. That is why we did not study the energy densities here but briefly summarized the observed final states. 


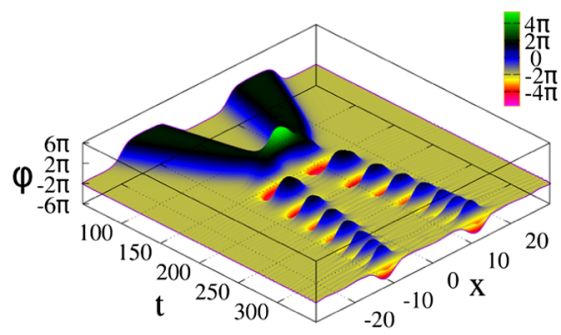

(a) $R=0.50$

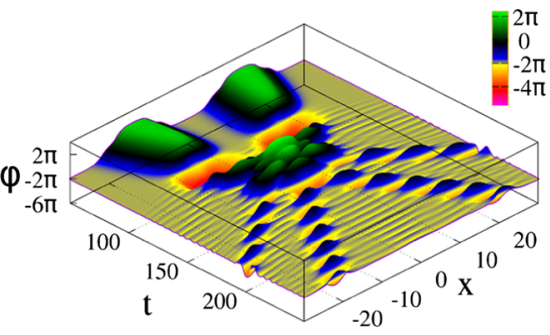

(d) $R=2.0$

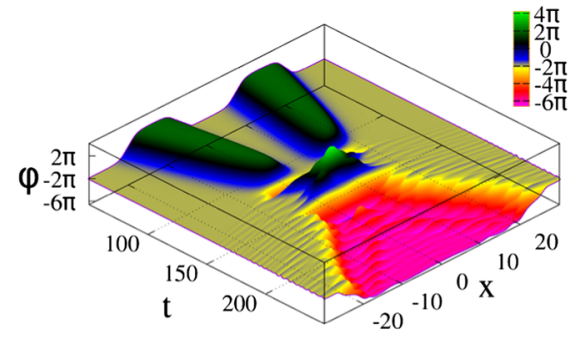

(b) $R=1.0$

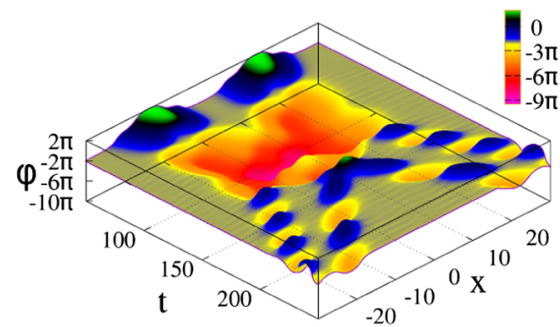

(e) $R=2.5$

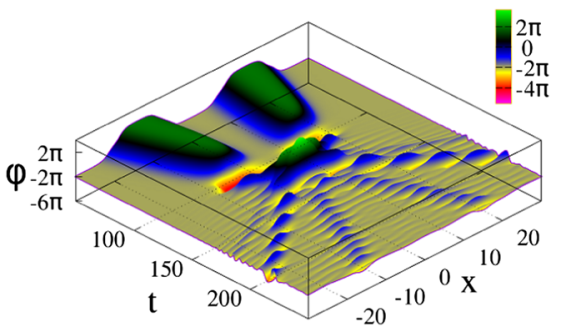

(c) $R=1.5$

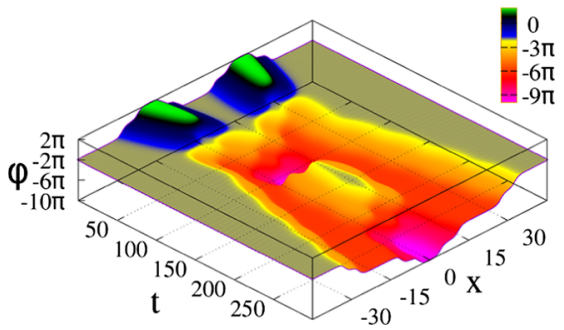

(f) $R=3.0$

Fig. 12 Space-time picture of collisions of four kinks for different $R$ 's

\section{Discussion and conclusion}

We have studied collisions of several kinks of the double sine-Gordon model in one point (in a small spatial region). We have performed numerical simulations of

(a) collisions of a kink and an antikink,

(b) collisions of two kinks and an antikink in one point,

(c) collisions of two kinks and two antikinks in one point.

Collisions of two kinks (i.e. kink-antikink scattering) are thoroughly investigated in literature [10,20,91]. In this paper we focused on energy distributions in the kink-antikink collisions at various values of the parameter $R$. We have obtained $R$-dependences of the maximal (over $x$ and $t$ ) kinetic, gradient, potential and total energy densities, Fig. 5. The maximal kinetic, gradient and total energy densities behave rather stochastic at $0.5 \lesssim R \lesssim 2.5$, while at $R \lesssim 0.5$ and $R \gtrsim 2.5$ smooth and monotonous segments are observed. We suppose that such behavior could be a consequence of the fact that the initial velocity of the kinks $v_{\text {in }}=0.1$ is less than the critical velocity $v_{\text {cr }}$, see [10]. This, in turn, leads to kinks' capture and formation of a bion. Energy redistribution in a bion is complicated and can be viewed as chaotic. At the same time, at $R \lesssim 0.5$ and $R \gtrsim 2.5$ we have $v_{\text {in }}>v_{\text {cr }}$, and colliding kinks bounce off each other after the first impact. In this case the complex energy redistribution does not take place, and $R$-dependences of the maximal energy densities are smooth and monotonous.
Besides, at $1.5 \lesssim R \lesssim 1.6$ we also observed small smooth and monotonous segments of the maximal energy densities in Fig. 5. In this range of the parameter, unlike other close values of $R$, in the final state we have two escaping oscillons formed after two oscillations of the kink-antikink bound state.

In three kinks collisions (in collisions of two kinks and an antikink) we observed various final states, in particular, similar to (i) excited kink ( $R=0.5$, Fig. 9a), (ii) excited kink and escaping oscillons ( $R=1.0,1.5,2.0,2.5$, Fig. 9b-e), and (iii) two escaping kinks and an antikink at rest $(R=3.0$, Fig. 9f). The maximal kinetic, gradient and total energy densities behave stochastic. At the same time, within the range $1.7 \lesssim R \lesssim 2.1$ we observed smooth and monotonous segments of the curves. We found that it could be a consequence of the formation of two escaping oscillons in the final state right after the kinks collision. Notice that the maximal total energy density in three-kink collisions is considerably larger than in two-kink collisions, see Figs. 5 and 10. At the same time, the maximal potential energy density is nearly the same for the two- and three-kink collisions. It means that the collision process does not affect on the maximal potential energy density. It is interesting that similar situation was observed in the multi-kink collisions in the sine-Gordon model [88] (up to seven kinks collision) and in the collisions of odd number of kinks in the $\varphi^{4}$ (up to five kinks collision) [89], while in the collisions of even number of kinks in the $\varphi^{4}$ (up to four kinks collision) and in the $\varphi^{6}$ multi-kink collisions the maximal potential energy depends on the number of colliding kinks $[87,89]$. 
Finally, we have studied collisions of four kinks (two kinks and two antikinks). The initial positions and velocities of the kinks were fit in such a way in order to provide almost simultaneous collision of all four waves in one point. After collision we observed a vast variety of the final states. Besides, the maximal energy densities strongly depend on the initial positions of the colliding kinks. That is why the analysis of the maximal energy densities requires detailed investigation of their dependences on parameters of the initial condition, which could be a subject of a separate publication. In this paper we have limited ourselves to the classification of the most typical final configurations. In particular, at $R=0.5$ in the final state we observed two oscillating structures which we classified as bound states of the DSG kink and antikink. At $R=1.0$ we observed escaping kink and antikink along with two oscillons - bound states of subkinks, which are components of the DSG kink (antikink). At $R=1.5$ and $R=2.0$ - only oscillons, at $R=3.0$ - escaping antikink and kink, while the other kink-antikink pair forms a bion localized near the collision point.

So we see a vast variety of final states in collisions of more than two kinks. Moreover, the behavior of the maximal energy densities strongly depends on oscillating structures being formed in the final state.

In conclusion, we would like to mention the following issues which are beyond the scope of our paper, nevertheless, in our opinion, could become subject to future work.

- First, the multi-kink scattering within the field-theoretic models with polynomial potentials of 8th or higher degrees. As we have already mentioned in the Introduction, such models can have kinks with long-range interaction between them due to the kinks' power-law tails. This long-range interaction could lead to new collective phenomena in multi-kink systems.

- Second, the multi-kink scattering within models with non-polynomial potentials, e.g., sinh-deformed $\varphi^{4}$ or $\varphi^{6}$, could be of interest, and will be studied in a separate publication.

Acknowledgements The work of MEPhI group was supported by the MEPhI Academic Excellence Project (Contract No. 02.a03.21.0005, 27.08.2013). V.A. Gani also acknowledges the support of the Russian Foundation for Basic Research under Grant No. 19-02-00971.

Data Availability Statement This manuscript has no associated data or the data will not be deposited. [Authors' comment: This is theoretical work, so we have not used any data.]

Open Access This article is distributed under the terms of the Creative Commons Attribution 4.0 International License (http://creativecomm ons.org/licenses/by/4.0/), which permits unrestricted use, distribution, and reproduction in any medium, provided you give appropriate credit to the original author(s) and the source, provide a link to the Creative Commons license, and indicate if changes were made. Funded by SCOAP . $^{3}$

\section{References}

1. R. Rajaraman, Solitons and Instantons: An Introduction to Solitons and Instantons in Quantum Field Theory (North-Holland, Amsterdam, 1982)

2. A. Vilenkin, E.P.S. Shellard, Cosmic Strings and Other Topological Defects (Cambridge University Press, Cambridge, 2000)

3. N. Manton, P. Sutcliffe, Topological Solitons (Cambridge University Press, Cambridge, 2004)

4. T. Vachaspati, Kinks and Domain Walls: An Introduction to Classical and Quantum Solitons (Cambridge University Press, Cambrifge, 2006)

5. V.A. Gani, A.E. Kudryavtsev, M.A. Lizunova, Kink interactions in the (1+1)-dimensional $\varphi^{6}$ model. Phys. Rev. D 89, 125009 (2014). arXiv: 1402.5903

6. V.A. Gani, V. Lensky, M.A. Lizunova, Kink excitation spectra in the $(1+1)$-dimensional $\varphi^{8}$ model. JHEP 08, 147 (2015). arXiv: 1506.02313

7. V.A. Gani, V. Lensky, M.A. Lizunova, E.V. Mrozovskaya, Excitation spectra of solitary waves in scalar field models with polynomial self-interaction. J. Phys. Conf. Ser. 675, 012019 (2016). arXiv:1602.02636

8. D. Bazeia, E. Belendryasova, V.A. Gani, Scattering of kinks of the sinh-deformed $\varphi^{4}$ model. Eur. Phys. J. C 78, 340 (2018). arXiv: 1710.04993

9. D. Bazeia, E. Belendryasova, V.A. Gani, Scattering of kinks in a non-polynomial model. J. Phys. Conf. Ser. 934, 012032 (2017). arXiv: 1711.07788

10. V.A. Gani, A.M. Marjaneh, A. Askari, E. Belendryasova, D. Saadatmand, Scattering of the double sine-Gordon kinks. Eur. Phys. J. C 78, 345 (2018). arXiv:1711.01918

11. E. Belendryasova, V.A. Gani, A.M. Marjaneh, D. Saadatmand, A. Askari, A new look at the double sine-Gordon kink-antikink scattering. J. Phys. Conf. Ser. 1205, 012007 (2019). arXiv:1810.00667

12. A.R. Gomes, F.C. Simas, K.Z. Nobrega, P.P. Avelino, False vacuum decay in kink scattering. JHEP 10, 192 (2018). arXiv:1805.00991

13. D. Bazeia, A.R. Gomes, K.Z. Nobrega, F.C. Simas, Kink scattering in a hybrid model. Phys. Lett. B 793, 26 (2019). arXiv:1805.07017

14. P. Dorey, T. Romańczukiewicz, Resonant kink-antikink scattering through quasinormal modes. Phys. Lett. B 779, 117 (2018). arXiv: 1712.10235

15. J.G.F. Campos, A. Mohammadi, Quasinormal modes in kinkantikink interactions: a toy model. arXiv:1905.00835

16. Y. Zhong, Z.-C. Jiang, Y.-X. Liu, Y.-Q. Wang, Collision of two kinks with inner structure. arXiv:1906.02920

17. D.K. Campbell, M. Peyrard, Solitary wave collisions revisited. Phys. D 18, 47 (1986)

18. T.I. Belova, A.E. Kudryavtsev, Solitons and their interactions in classical field theory. Phys. Usp. 40, 359 (1997)

19. T.I. Belova, A.E. Kudryavtsev, Solitons and their interactions in classical field theory. Usp. Fiz. Nauk 167, 377 (1997)

20. V.A. Gani, A.E. Kudryavtsev, Kink-antikink interactions in the double sine-Gordon equation and the problem of resonance frequencies. Phys. Rev. E 60, 3305 (1999). arXiv:cond-mat/9809015

21. P. Dorey, K. Mersh, T. Romańczukiewicz, Y. Shnir, Kink-antikink collisions in the $\phi^{6}$ model. Phys. Rev. Lett. 107, 091602 (2011). arXiv: 1101.5951

22. E. Belendryasova, V.A. Gani, Scattering of the $\varphi^{8}$ kinks with power-law asymptotics. Commun. Nonlinear Sci. Numer. Simul. 67, 414 (2019). arXiv:1708.00403

23. E. Belendryasova, V.A. Gani, Resonance phenomena in the $\varphi^{8}$ kinks scattering. J. Phys. Conf. Ser. 934, 012059 (2017). arXiv: 1712.02846

24. A.R. Gomes, R. Menezes, J.C.R.E. Oliveira, Highly interactive kink solutions. Phys. Rev. D 86, 025008 (2012). arXiv:1208.4747 
25. R.V. Radomskiy, E.V. Mrozovskaya, V.A. Gani, I.C. Christov, Topological defects with power-law tails. J. Phys. Conf. Ser. 798, 012087 (2017). arXiv: 1611.05634

26. I.C. Christov, R.J. Decker, A. Demirkaya, V.A. Gani, P.G. Kevrekidis, R.V. Radomskiy, Long-range interactions of kinks. Phys. Rev. D 99, 016010 (2019). arXiv: 1810.03590

27. I.C. Christov, R.J. Decker, A. Demirkaya, V.A. Gani, P.G. Kevrekidis, A. Khare, A. Saxena, Kink-kink and kink-antikink interactions with long-range tails. Phys. Rev. Lett. 122, 171601 (2019). arXiv: 1811.07872

28. N.S. Manton, Force between kinks with long-range tails. arXiv: 1810.00788

29. N.S. Manton, Forces between kinks and antikinks with long-range tails. J. Phys. A Math. Theor. 52, 065401 (2019). arXiv:1810.03557

30. D. Bazeia, R. Menezes, D.C. Moreira, Analytical study of kinklike structures with polynomial tails. J. Phys. Commun. 2, 055019 (2018). arXiv:1805.09369

31. I. Takyi, H. Weigel, Collective coordinates in one-dimensional soliton models revisited. Phys. Rev. D 94, 085008 (2016). arXiv: 1609.06833

32. A. Demirkaya, R. Decker, P.G. Kevrekidis, I.C. Christov, A. Saxena, Kink dynamics in a parametric $\phi^{6}$ system: a model with controllably many internal modes. JHEP 12, 071 (2017). arXiv: 1706.01193

33. J.K. Perring, T.H.R. Skyrme, A model unified field equation. Nucl. Phys. 31, 550 (1962)

34. R. Rajaraman, Intersoliton forces in weak-coupling quantum field theories. Phys. Rev. D 15, 2866 (1977)

35. N.S. Manton, An effective Lagrangian for solitons. Nucl. Phys. B 150, 397 (1979)

36. P.G. Kevrekidis, A. Khare, A. Saxena, Solitary wave interactions in dispersive equations using Manton's approach. Phys. Rev. E 70, 057603 (2004). arXiv:nlin/0410045

37. V.A. Gani, A.E. Kudryavtsev, Collisions of domain walls in a supersymmetric model. Phys. Atom. Nucl. 64, 2043 (2001). arXiv:hep-th/9904209

38. V.A. Gani, A.E. Kudryavtsev, Collisions of domain walls in a supersymmetric model. Yad. Fiz. 64, 2130 (2001). arXiv:hep-th/9912211

39. V.A. Lensky, V.A. Gani, A.E. Kudryavtsev, Domain walls carrying a U(1) charge. Sov. Phys. JETP 93, 677 (2001). arXiv:hep-th/0104266

40. V.A. Lensky, V.A. Gani, A.E. Kudryavtsev, Domain walls carrying a U(1) charge. Zh. Eksp. Teor. Fiz. 120, 778 (2001). arXiv:hep-th/0104266

41. A. Alonso Izquierdo, M.A.G. Leon, J.M. Guilarte, The Kink variety in systems of two coupled scalar fields in two space-time dimensions. Phys. Rev. D 65, 085012 (2002). arXiv:hep-th/0201200

42. V.A. Gani, N.B. Konyukhova, S.V. Kurochkin, V.A. Lensky, Study of stability of a charged topological soliton in the system of two interacting scalar fields. Comput. Math. Math. Phys. 44, 1968 (2004). arXiv:0710.2975

43. V.A. Gani, N.B. Konyukhova, S.V. Kurochkin, V.A. Lensky, Study of stability of a charged topological soliton in the system of two interacting scalar fields. Zh. Vychisl. Mat. Mat. Fiz. 44, 2069 (2004). arXiv:0710.2975

44. A. Alonso Izquierdo, D. Bazeia, L. Losano, J. Mateos Guilarte, New models for two real scalar fields and their kink-like solutions. Adv. High Energy Phys. 2013, 183295 (2013). arXiv:1308.2724

45. A. Alonso-Izquierdo, J.M. Guilarte, Quantum-induced interactions in the moduli space of degenerate BPS domain walls. J. High Energy Phys. 01, 125 (2014). arXiv: 1307.0740

46. H. Katsura, Composite-kink solutions of coupled nonlinear wave equations. Phys. Rev. D 89, 085019 (2014). arXiv:1312.4263
47. A. Alonso Izquierdo, Kink dynamics in a system of two coupled scalar fields in two space-time dimensions. Phys. D 365, 12 (2018). arXiv: 1711.08784

48. A. Alonso Izquierdo, Reflection, transmutation, annihilation, and resonance in two-component kink collisions. Phys. Rev. D 97, 045016 (2018). arXiv:1711.10034

49. A. Alonso Izquierdo, Kink dynamics in the MSTB Model. Phys. Scr. 94, 085302 (2019). arXiv:1804.05605

50. A. Alonso Izquierdo, Asymmetric kink scattering in a twocomponent scalar field theory model. Commun. Nonlinear Sci. Numer. Simul. 75, 200 (2019). arXiv:1901.03089

51. J.R. Morris, Interacting kinks and meson mixing. Ann. Phys. 400, 346 (2019). arXiv: 1901.01467

52. A. Alonso Izquierdo, Non-topological kink scattering in a twocomponent scalar field theory model. arXiv: 1906.05040

53. M. Mohammadi, N. Riazi, The affective factors on the uncertainty in the collisions of the soliton solutions of the double field sineGordon system. Commun. Nonlinear Sci. Numer. Simul. 72, 176 (2019). arXiv: 1906.07498

54. V.A. Gani, M.A. Lizunova, R.V. Radomskiy, Scalar triplet on a domain wall: an exact solution. JHEP 04, 043 (2016). arXiv: 1601.07954

55. V.A. Gani, M.A. Lizunova, R.V. Radomskiy, Scalar triplet on a domain wall. J. Phys. Conf. Ser. 675, 012020 (2016). arXiv: 1602.04446

56. N. Blyankinshtein, Q-lumps on a domain wall with a spin-orbit interaction. Phys. Rev. D 93, 065030 (2016). arXiv:1510.07935

57. V.A. Gani, V.G. Ksenzov, A.E. Kudryavtsev, Example of a selfconsistent solution for a fermion on domain wall. Phys. Atom. Nucl. 73, 1889 (2010). arXiv:1001.3305

58. V.A. Gani, V.G. Ksenzov, A.E. Kudryavtsev, Example of a selfconsistent solution for a fermion on domain wall. Yad. Fiz. 73, 1940 (2010). arXiv: 1001.3305

59. V.A. Gani, V.G. Ksenzov, A.E. Kudryavtsev, Stable branches of a solution for a fermion on domain wall. Phys. Atom. Nucl. 74, 771 (2011). arXiv: 1009.4370

60. V.A. Gani, V.G. Ksenzov, A.E. Kudryavtsev, Stable branches of a solution for a fermion on domain wall. Yad. Fiz. 74, 797 (2011). arXiv: 1009.4370

61. Y. Brihaye, A. Cisterna, B. Hartmann, G. Luchini, From topological to nontopological solitons: Kinks, domain walls, and Q-balls in a scalar field model with a nontrivial vacuum manifold. Phys. Rev. D 92, 124061 (2015). arXiv:1511.02757

62. A.Yu. Loginov, Q kink of the nonlinear $\mathrm{O}(3) \sigma$ model involving an explicitly broken symmetry. Phys. Atom. Nucl. 74, 740 (2011)

63. A.Yu. Loginov, Q kink of the nonlinear $\mathrm{O}(3) \sigma$ model involving an explicitly broken symmetry. Yad. Fiz. 74, 766 (2011)

64. D. Bazeia, A. Mohammadi, Fermionic bound states in distinct kinklike backgrounds. Eur. Phys. J. C 77, 203 (2017). arXiv:1702.00891

65. D. Bazeia, A. Mohammadi, D.C. Moreira, Fermion bound states in geometrically deformed backgrounds. Chin. Phys. C 43, 013101 (2019). arXiv:1706.04406

66. M. Mai, P. Schweitzer, Energy momentum tensor, and the D-term of Q-balls. Phys. Rev. D 86, 076001 (2012). arXiv:1206.2632

67. M. Mai, P. Schweitzer, Radial excitations of Q-balls, and their Dterm. Phys. Rev. D 86, 096002 (2012). arXiv:1206.2930

68. M. Cantara, M. Mai, P. Schweitzer, The energy-momentum tensor and D-term of Q-clouds. Nucl. Phys. A 953, 1 (2016). arXiv: 1510.08015

69. I.E. Gulamov, E.Ya. Nugaev, M.N. Smolyakov, Analytic Q-ball solutions and their stability in a piecewise parabolic potential. Phys. Rev. D 87, 085043 (2013). arXiv: 1303.1173

70. D. Bazeia, M.A. Marques, R. Menezes, Exact solutions, energy and charge of stable Q-balls. Eur. Phys. J. C 76, 241 (2016). arXiv:1512.04279 
71. D. Bazeia et al., Compact Q-balls. Phys. Lett. B 758, 146 (2016). arXiv: 1604.08871

72. D. Bazeia, L. Losano, M.A. Marques, R. Menezes, Split Q-balls. Phys. Lett. B 765, 359 (2017). arXiv: 1612.04442

73. D. Levkov, E. Nugaev, A. Popescu, The fate of small classically stable Q-balls. JHEP 12, 131 (2017). arXiv:1711.05279

74. V. Loiko, I. Perapechka, Ya. Shnir, Q-balls without a potential. Phys. Rev. D 98, 045018 (2018). arXiv:1805.11929

75. A. Kovtun, E. Nugaev, A. Shkerin, Vibrational modes of Q-balls. Phys. Rev. D 98, 096016 (2018). arXiv: 1805.03518

76. A.Yu. Loginov, A two-dimensional soliton system of vortex and Q-ball. Phys. Lett. B 777, 340 (2018). arXiv:1901.00993

77. A.Yu. Loginov, One-dimensional soliton system of gauged Q-ball and anti-Q-ball. Phys. Rev. D 99, 065011 (2019). arXiv: 1901.00272

78. E. Greenwood, E. Halstead, R. Poltis, D. Stojkovic, Electroweak vacua, collider phenomenology, and possible connection with dark energy. Phys. Rev. D 79, 103003 (2009). arXiv:0810.5343

79. D.C. Valle, E.W. Mielke, Relativistic soliton collisions of axion type dark matter. Phys. Lett. B 758, 93 (2016)

80. Y. Matsui, K. Horiguchi, D. Nitta, S. Kuroyanagi, Improved calculation of the gravitational wave spectrum from kinks on infinite cosmic strings. JCAP 11, 005 (2016). arXiv: 1605.08768

81. J.M. Wachter, K.D. Olum, Gravitational smoothing of kinks on cosmic string loops. Phys. Rev. Lett. 118, 051301 (2017). arXiv: 1609.01153

82. J. Ye, K. Wang, Y.F. Cai, Superconducting cosmic strings as sources of cosmological fast radio bursts. Eur. Phys. J. C 77, 720 (2017). arXiv: 1705.10956

83. C. Ringeval, T. Suyama, Stochastic gravitational waves from cosmic string loops in scaling. JCAP 12, 027 (2017). arXiv:1709.03845
84. V.A. Gani, A.A. Kirillov, S.G. Rubin, Classical transitions with the topological number changing in the early Universe. JCAP 04, 042 (2018). arXiv: 1704.03688

85. V.A. Gani, A.A. Kirillov, S.G. Rubin, Transitions between topologically non-trivial configurations. J. Phys. Conf. Ser. 934, 012046 (2017). arXiv: 1711.07700

86. D. Saadatmand, S.V. Dmitriev, P.G. Kevrekidis, High energy density in multisoliton collisions. Phys. Rev. D 92, 056005 (2015). arXiv: 1506.01389

87. A.M. Marjaneh, V.A. Gani, D. Saadatmand, S.V. Dmitriev, K. Javidan, Multi-kink collisions in the $\phi^{6}$ model. JHEP 07, 028 (2017). arXiv: 1704.08353

88. A.M. Marjaneh, A. Askari, D. Saadatmand, S.V. Dmitriev, Extreme values of elastic strain and energy in sine-Gordon multi-kink collisions. Eur. Phys. J. B 91, 22 (2017). arXiv:1710.10159

89. A.M. Marjaneh, D. Saadatmand, K. Zhou, S.V. Dmitriev, M.E. Zomorrodian, High energy density in the collision of $N$ kinks in the $\phi^{4}$ model. Commun. Nonlinear Sci. Numer. Simul. 49, 30 (2017). arXiv: 1605.09767

90. A. Askari, D. Saadatmand, S.V. Dmitriev, K. Javidan, High energy density spots and production of kink-antikink pairs in particle collisions. Wave Motion 78, 54 (2018). arXiv:1608.01847

91. D.K. Campbell, M. Peyrard, P. Sodano, Kink-antikink interactions in the double sine-Gordon equation. Phys. D 19, 165 (1986)

92. B.A. Malomed, Dynamics and kinetics of solitons in the driven damped double sine-Gordon equation. Phys. Lett. A 136, 395 (1989)

93. S. Nazifkar, K. Javidan, Collective coordinate analysis for double sine-Gordon model. Braz. J. Phys. 40, 102 (2010) 